\title{
A Local-Global Approach to Solving Ideal Lattice Problems
}

\author{
Tian Yuan ${ }^{1}$, Sun Rongxin, Zhu Xueyong, and Cai Wuyang \\ Network Department, Software School, Dalian University of Technology, P.R.China, \\ tianyuan_ca@sina.com
}

\begin{abstract}
We construct an innovative SVP(CVP) solver for ideal lattices in case of any relative extension of number fields $L / K$ of degree $n$ where $L$ is real(contained in $R$ ). The solver, by exploiting the relationships between the so-called local and global number fields, reduces solving $\operatorname{SVP}(\mathrm{CVP})$ of the input ideal $A$ in field $L$ to solving a set of (at most $n) \mathrm{SVP}(\mathrm{CVP})$ of the ideals $A_{i}$ in field $L_{i}$ with relative degree $1 \leq n_{i}<n$ and $\sum_{i} n_{i}=n$. The solver's space-complexity is polynomial and its time-complexity's explicit dependence on the dimension (relative extension degree $n$ ) is also polynomial. More precisely, our solver's time-complexity is $\operatorname{poly}\left(n,|S|, N_{P G}, N_{P T}, N_{d}, N_{l}\right)$ where $|S|$ is bit-size of the input data and $N_{P G}, N_{P T}, N_{d}, N_{l}$ are the number of calls to some oracles for relatively simpler problems (some of which are decisional). This feature implies that if such oracles can be implemented by efficient algorithms (with time-complexity polynomial in $n$ ), which is indeed possible in some situations, our solver will perform in this case with timecomplexity polynomial in $n$. Even if there is no efficient implementations for these oracles, this solver's time-complexity may still be significantly lower than those for general lattices, because these oracles may be implemented by algorithms with sub-exponential time-complexity
\end{abstract}

Keywords:Shortest Vector Problem(SVP); Closest Vector Problem(CVP); ideal lattices; field valuations; non-Archimedean valuations; local field; global field.

\section{Introduction}

Lattice problems take important roles in combinatorial optimization, public-key cryptography and many other fields in computer science[1-5]. In the shortest lattice vector problem (SVP), a non-zero lattice vector $\boldsymbol{x}$ in $\mathbf{B Z}^{n}$ is to be found to minimize $|\boldsymbol{x}|$ on input the lattice basis matrix $\mathbf{B}$ with respect to some specific norm $\|$ in $R^{n}$. In the closest lattice vector problem (CVP), a lattice vector $\boldsymbol{x}$ is to be found to minimize $|\boldsymbol{u}-\boldsymbol{x}|$ on input the basis matrix $\mathbf{B}$ and a target vector $\boldsymbol{u}$ in $R^{n}$. In recent years, lots of innovative cryptographic schemes and protocols have been devised with proofs of security under the assumption that there is not (probabilistic and sometimes quantum) polynomial-time algorithm to solve arbitrary instances of variants of SVP and CVP.

From a computational hardness perspective, SVP, CVP and other related variants are NP-hard under deterministic (e.g.,CVP) or randomized (e. g.,SVP) reductions [4]. Even some approximation variants of these problems are proven to be NP-hard if the approximation factor is within some specific range. Despite of these facts, finding new algorithms to solve lattice problems exactly are still interesting and meaningful both because many applications (e.g., in mathematics and communication theory) involve lattices in relatively small dimensions, and 
because approximation algorithms for high dimensional lattices for which the exact solution is infeasible typically involve the exact solution of low dimensional sub-problems.

Recently a sub-category of lattices, the ideal lattice, is discovered to have indispensible values in innovative cryptography applications, e. g., the wonderful fully homomorphic encryption scheme for secure cloud computing[2], stimulating lots of works in cryptography theory and practices. On the one hand, such schemes are based-on some computational hardness hypothesis on some problems in ideal lattices, e.g., SVP or CVP's hardness, on the other hand, few deep knowledge is known on these points. Since the ideal lattice has plenty of rich intrinsic algebraic properties the general lattice doesn't have, it's reasonable to ask whether its related problems, e.g., SVP and CVP, are really as hard as those of general lattices, or "how easy" are they in comparison with their counterparts in general lattices? No matter what the answer (positive or negative) to this question would be, it will have fundamental significance to ideal lattice theory and applications.

In this paper we work on this question in case of SVP and CVP problems in an algorithmic approach.

Roadmap In this paper we construct the generic algorithms for exactly solving SVP and CVP of ideal lattices by exploiting the algebraic properties uniquely owned by ideal lattices. Sect.2 briefly summarizes the current SVP and CVP solvers for general lattices and an overview on the innovations in our work. Sect.3 provide necessary foundations for our constructions. For logic clearness, we provide a high level algorithm description in Sect.4 at first and then present all low level technical details in Sect.5. Sect.6 concludes and points out some future works.

\section{Related Works}

To find the exact solution to lattice problems, so far three main families of SVP and CVP solvers exist which are listed in Table 1. With our knowledge, there're no generic algorithms for ideal lattice problems, except some ones modified from the solvers for non-ideal lattices which doesn't essentially exploit the ideal lattice's algebraic properties.

Among the solvers in Table 1, MV and Kannan algorithms are deterministic while AKS algorithms are randomized. All algorithms work in $\ell^{2}$-norm (AKS algorithm can work in other norms, e.g., $\left.\ell_{\infty}\right)$. The core of MV algorithm[6] is to compute the Voronoi cell of the lattice[1], whose knowledge facilitates the tasks to solve SVP and CVP. Kannan algorithm[7] relies on a deterministic procedure to enumerate all lattice vectors below a prescribed norm or within a prescribed distance to the target vector. This procedure uses the Grahm-Schmidt orthogonalization of the input lattice basis to recursively bound the integer coordinates of the candidate solutions. The AKS algorithm[8] is the first single-exponential time (random) algorithm for SVP.Recently this algorithm has been significantly improved and the currently best time complexity is $2^{2.465 n+o(n)}[3]$. However, the AKS variant solver for CVP only finds the $(1+\varepsilon)$-approximate solution for arbitrary $\varepsilon>0$ in time complexity bounded by $(2+1 / \varepsilon)^{O(n)}[9,10]$.

It's already known that when the lattice dimension $n$ is fixed, there are polynomial time-complicated solvers for lattice problems, e.g.,SVP/CVP. i.e., lattice problem's computational hardness only depends on dimension $n$ (Tab.1).

Some related works show that there are important differences in computational complexity between the lattice problems of general and ideal lattices. For 
example, some decisional problems of the ideal lattice family with constant root discriminant is in $P$ while the counterparts of general lattice are NP-hard[11]. However, (with our knowledge) there is not search or optimization SVP/CVP (see the concepts in Sect.3.1) solver exploiting the ideal lattice algebraic features and performing significantly better than the best known solvers for general lattices.

Overview on innovations of our approach: construction and performance

Our algorithms constructed in this paper are to find the exact solutions to SVP and CVP in ideal lattices.In this paper we only deal with the case of real number field, i. e., the number field which the input ideal belongs to is contained in $R$.

Our solver works on the input $(L / K, A)$ where $L / K$ is a finite-degree extension of number field with degree $n, A$ is an (fractional) ideal in $L, K$ is fixed and $(L, A)$ is arbitrarily given. In other words, our solver can work for any finite-degree relative extension, not only the special case of $L / Q$ (where $Q$ is the rational number field).

In construction aspects, our solver, by exploiting the relationships between the so-called local and global number fields, reduces solving SVP(CVP) of the input ideal $A$ in field $L$ to solving a set of (at most $n) \operatorname{SVP}(\mathrm{CVP}$ ) of the ideals $A_{i}$ in field $L_{i}$ with relative degree $1 \leq n_{i}<n$ and $\sum_{i} n_{i}=n$. Roughly speaking, by tensor-producting $L$ with a local field $K_{P}$ where $P$ is an appropriately selected (not unique) prime ideal in the ground field $K$, the tensor product (as a $n$-dimensional vector space on the local field $K_{P}$ ) can be always decomposed into a set of sub-spaces of dimension $n_{i}<n$ which are orthogonal each other and $\sum_{i} n_{i}=n$. Furthermore, this orthogonal decomposition is metric-preserving and by constructing appropriate injective homomorphisms all operations in intermediate local fields can be replaced by those in some intermediate global fields(i. e., ordinary number fields), so that the solution to the original problem can be effectively reconstructed from the solutions to the sub-problems. This procedure can proceed recursively down to a set of (at most $n$ ) sub-problems of ideal lattices with dimensions as low as possible. In particular, in case of Galois extension $L / K$, each recursion can decrease the problem's dimension by at least half.

Table 1: The existed families of SVP and CVP solvers for general lattices

\begin{tabular}{cccl}
\hline Solvers & $\begin{array}{c}\text { Time complexity } \\
\text { upper bound }\end{array}$ & $\begin{array}{c}\text { Space complexity } \\
\text { upper bound }\end{array}$ & Remarks \\
\hline \hline Kannan & $n^{O(n)}$ & poly $(n)$ & $\begin{array}{l}\text { deterministic; } \\
\text { the O-constant is } \\
\text { improved as small as } 1 / 2 e\end{array}$ \\
{$[3,7,12]$} & & $2^{O(n)}$ & deterministic \\
\hline $\mathrm{MV}[3,6]$ & $2^{2 n+o(n)}$ & SVP: $2^{1.325 n+o(n)}$ & randomized; \\
\hline $\mathrm{AKS}$ & $\mathrm{SVP}: 2^{2.465 n+o(n)}$ & $\mathrm{CVP}$ only \\
\hline $3,8-10]$ & $\mathrm{CVP}:(2+1 / \varepsilon)^{O(n)}$ & $\mathrm{CVP}:(1+1 / \varepsilon)^{O(n)}$ & solves $(1+\varepsilon)$-CVP $\left(1+{ }^{\circ}\right.$
\end{tabular}

In performance aspects, our $\operatorname{SVP}(\mathrm{CVP})$ solver's space-complexity is polynomial. Its time-complexity's explicit dependence on the dimension (relative extension degree $n$ of the number fields) is also polynomial. More precisely, our 
solver's time-complexity is

$$
\operatorname{poly}\left(n,|S|, N_{P G}, N_{P T}, N_{d}, N_{l}\right)
$$

where $|S|$ is bit-size of the input data and $N_{P G}, N_{P T}, N_{d}, N_{l}$ are the number of calls to the oracles(Sect.5.5) for some relatively simpler problems (some of them are decisional, e. g., ideal's primality testing in the extended field $L$ ). This feature implies that if such oracles can be implemented by efficient algorithms (with time-complexity polynomial in $n$ ), which is really possible in some situations, our solver will perform in this case with time-complexity polynomial in $n$. Even there is no efficient implementations for these oracles, this solver's time-complexity may still be significantly lower than those for general lattices (e. g., Tab.1), because their implementations may be only sub-exponential in time-complexity.

\section{Preliminaries}

In this section we present all basic notions and facts fundamental to our work in this paper. For more details we refer readers to [4](for general theory on lattices), [13-16](for algebraic number theory) and [17] (for abstract algebra, e. g., the general notions and facts on (Detekind) rings, ideals, unique factorization domains, fields and Galois theory).

\subsection{Lattices, SVP and CVP}

The set of rational integers is denoted by $Z$ and rational numbers by $Q$. A lattice is a finitely generated discrete subset in Euclidean space. More explicitly, in the Euclidean space $R^{n}$ with a positive non-singular bilinear form $\left.<_{\text {... }}\right\rangle$, a $n$-dimensional rational lattice, denoted $\Lambda(\mathbf{B})$ where $\mathbf{B}$ is a matrix of rank $n$ with column vectors $\left(\boldsymbol{b}_{1}, \ldots, \boldsymbol{b}_{n}\right)$, is the set of vectors $\left\{x_{1} \boldsymbol{b}_{1}+\ldots+x_{n} \mathbf{b}_{n}: x_{1}, \ldots, x_{n} \in\right.$ $Z\}$ where the values $\left\langle\boldsymbol{b}_{i}, \boldsymbol{b}_{j}\right\rangle$ are all rational numbers. The lattice with basis $\boldsymbol{b}_{1}, \ldots, \boldsymbol{b}_{n}$ is denoted $Z \boldsymbol{b}_{1}+\ldots+Z \boldsymbol{b}_{n}$. A lattice is called integral if $\left\langle\boldsymbol{b}_{i}, \boldsymbol{b}_{j}\right\rangle$ are all integers.

For any vector $\boldsymbol{u}=\left(u_{1}, \ldots, u_{n}\right)$ in $R^{n}$, its norm $\langle\boldsymbol{u}, \boldsymbol{u}\rangle^{1 / 2}$ is denoted $|\boldsymbol{u}|$. It's easy to verify that the squared norm of any lattice vector in an integral lattice is always an integer.

Lattice Problems Given a lattice $\Lambda(\mathbf{B})=Z \boldsymbol{b}_{1}+\ldots+Z \boldsymbol{b}_{n}$, let

$$
\lambda_{1}(\Lambda) \equiv \min \{|\boldsymbol{x}|: \boldsymbol{x} \text { in } \Lambda \text { and non-zero }\}
$$

be the minimal value of the norms of non-zero lattice vectors in $\Lambda(\mathbf{B})$. The optimization shortest vector problem with respect to the norm $\|$ is to find $\lambda_{1}(\Lambda)$. The search shortest vector problem is to find a lattice vector $\boldsymbol{x}$ in $\Lambda$ such that $|\boldsymbol{x}|=\lambda_{1}(\Lambda)$. Given a lattice $\Lambda(\mathbf{B})$ and a rational target vector $\boldsymbol{u}$ in $Q^{n}$, let

$$
\operatorname{dist}(\Lambda ; \boldsymbol{u}) \equiv \min \{|\boldsymbol{x}-\boldsymbol{u}|: \boldsymbol{x} \text { in } \Lambda\}
$$

be the minimum distance between $\boldsymbol{u}$ and all lattice vectors in $\Lambda$. The optimization closest vector problem with respect to the norm $\|$ is to find $\operatorname{dist}(\Lambda ; \boldsymbol{u})$. The search closest vector problem is to find a lattice vector $\boldsymbol{x}$ in $\Lambda$ such that $|\boldsymbol{x}-\boldsymbol{u}|=$ $\operatorname{dist}(\Lambda ; \boldsymbol{u})$. 
There are many other lattice-related problems[18, 19]. For example, the covering radius of a lattice, $\mu(\Lambda)$, is defined as the maximal distance between any vector and the lattice. The covering radius problem is to find

$$
\mu(\Lambda) \equiv \max \left\{\operatorname{dist}(\Lambda ; \boldsymbol{u}): \boldsymbol{u} \text { in } Q^{n}\right\}
$$

In this paper we focus on the algorithms to solve SVP and CVP. It has been known that these problems are computationally hard[4, 19].We focus on constructing the algorithms for SVP and CVP (both in optimization and search version) for ideal lattices, a sub-category of the general lattices with rich algebraic structures originating from number theory.

\subsection{Number Fields and Ideal Lattices}

A number field $K=Q(\alpha)$ is an extension of the rational number field $Q$, by adding a root $\alpha$ of a polynomial $f(x) \in Z[x] . f(x)$ is called $\alpha$ 's minimal polynomial if it has the minimal degree among the polynomials in $Z[x]$ with $\alpha$ as a root. Such a polynomial is unique up to a constant factor in $Z$ and is always prime (irreducible) in $Z[x]$. The minimal polynomial $f(x)$ 's degree is called the degree of field extension $K / Q$ and denoted by $[K: Q]$.

As a subset in the real $(R)$ or complex $(C)$ number field, the arithmetic operations $a \pm b, a b, a / b$ in $K$ can be simply understood as the operations in $R$ or $C$. Another helpful and equivalent (isomorphic) picture is to regard $K$ as the quotient set of $K[x] /(f(x))$ with its arithmetic operations as polynomial addition, subtraction and multiplication modulo $f(x)$ (dividing by $g(x) \neq 0 \bmod f(x)$ equals multiplying by $G(x)$ where $G(x) g(x)=1 \bmod f(x))$. In this setting, the generator $\alpha$ in $K$ corresponds to $x$ in $K[x] /(f(x))$.

As in the general theory on finite-degree field extension, the number field $K$ can be regarded as a vector space on the ground field $Q$ in dimension $n=[K: Q]$. As a result, we can introduce the trace and norm to any element in the number field $K$, i. e., for any $z$ in $K$ we consider the linear operator $T(z) x=z x: K \rightarrow K$ derived by $z$ and define $\operatorname{Tr}(z)$ and $N(z)$ as the trace and determinant of $T(z)$ respectively. An important fact is that both $\operatorname{Tr}(z)$ and $N(z)$ fall in $Q$, and both of them fall in $Z$ when $z$ is in $O_{K}$ which is defined in sequel.

Within the number field $K$, there is an important subset, called $K$ 's integral ring, defined as

$O_{K} \equiv\left\{z\right.$ in $K:$ there exist $a_{0}, \ldots, a_{n-1}$ in $Z$ such that $\left.a_{0}+a_{1} z+\ldots+a_{n-1} z^{n-1}+z^{n}=0\right\}$

$O_{K}$ is a ring with the following properties[14-17]:

(1) $K$ is $O_{K}$ 's fractional field, i. e., for any element $u$ in $K$ there is $a$ and $b$ in $O_{K}$ such that $u=a / b$.

(2)For an (integral) ideal $A$ in $O_{K}$ (including $O_{K}$ itself), there always exist a set of $Z$-linear independent elements $\omega_{1}, \ldots, \omega_{n}$ in $A$ where $n=[K: Q]$, called $A$ 's integral basis, such that every element in $A$ can be uniquely represented as a $Z$-coefficient linear combination on $\omega_{1}, \ldots, \omega_{n}$. This fact is denoted as $A=$ $Z \omega_{1}+\ldots+Z \omega_{n}$.

For $O_{K}$, such basis is called $K$ 's integral basis and the determinant of the matrix $\operatorname{Tr}\left(\omega_{i} \omega_{j}\right)$, denoted $d_{K}$, is called the determinant of $K$, which is one of the most important invariant of $K$.

(3)For number field $K$, there are exactly $n=[K: Q]$ field embeddings (injective homomorphisms) mapping $K$ into $C$ which are fixed in $Q$ element-wise, among which $\rho_{1}, \ldots, \rho_{r_{1}}$ embed $K$ into $R$ and the other $2 r_{2}$ ones $\tau_{1}, \ldots, \tau_{2 r_{2}}$ 
(where each $\tau_{j}$ is complex conjugate to $\tau_{j+r_{2}}$ ) embed $K$ into $C$. Now comes one of the central facts and notions in this paper:

Any ideal $A$ is a discrete and finitely-generated subset in $R^{r_{1}} \times C^{r_{2}}$ by mapping $z$ in $A$ to $\left(\rho_{1}(z), \ldots, \rho_{r_{1}}(z)\right.$,

$\left.\tau_{1}(z), \ldots, \tau_{2 r_{2}}(z)\right)$ in $R^{r_{1}} \times C^{r_{2}}$, or equivalently by mapping $z$ to the real vector $\left(\rho_{1}(z), \ldots, \rho_{r_{1}}(z), \operatorname{Re}_{1}(z), \ldots\right.$,

$\left.\operatorname{Re} \tau_{r_{2}}(z), \operatorname{Im} \tau_{1}(z), \ldots, I m \tau_{r_{2}}(z)\right)$ in $R^{n}$. As a result, the ideal $A$ can be embedded into $R^{n}$ as a lattice of dimension $n$. When associating $A$ with the positive-definite and non-degenerate bilinear form

$$
<x, y>\equiv \operatorname{Tr}(x \bar{y}) \text { where } \bar{y} \text { denotes } y^{\prime} \text { s complex conjugation }
$$

we call $A$ (strictly speaking, its image under the above embedding) an ideal lattice. In consequence, we can setup the same problems as in Sect.3.1, e.g., SVP and CVP for ideal lattices.

It's easy to see that for any ideal $A$ in $O_{K}$, the ideal lattice is always integral.

(4)For any ideal $A$ in $O_{K}$, the quotient ring $O_{K} / A$ is always finite and its cardinality is denoted $N(A)$. In fact, let $A=Z \omega_{1}+\ldots+Z \omega_{n}$, then $N(A)^{2} d_{K}=$ $\operatorname{det}\left(\operatorname{Tr}\left(\omega_{i} \omega_{j}\right)\right)=$ the squared volume of the fundamental domain in the ideal lattice $A$.

(5)An ideal $P\left(\neq O_{K}\right)$ is called prime, if $x y$ in $P$ then always at least one of $x$ and $y$ is in $P$. For number field $K$, every prime ideal $P$ in $O_{K}$ is maximal, i. e., it is not contained in any ideal other than $O_{K}$.

Prime ideals are construction stones for number field arithmetic. For any prime ideal $P$, the quotient ring $O_{K} / P$ is actually always a finite field with cardinality $p^{f}$ where $p$ is a prime integer and $(p) \equiv p Z=P \cap Z$. As a result, $N(A)=p^{f}$ and $O_{K} / P$ is a $f$-degree extension of $F_{p}$.

(6)Every ideal $A$ in $O_{K}$ can be uniquely decomposed as the multiplication of a finite set of prime ideals, i. e., for any ideal $A$ there exist finite prime ideals $P_{1}, \ldots, P_{N}$ and positive rational integers $e_{1}, \ldots, e_{N}$ such that (in the sense of ideals multiplication)

$$
A=P_{1}^{e_{1}} \ldots P_{N}^{e_{N}}
$$

where both $P_{1}, \ldots, P_{N}$ and $e_{1}, \ldots, e_{N}$ are uniquely determined by $A$.

(7)For a subset $A$ in $K$, if $a \pm b$ is always in $A$ for both $a, b$ in $A$ and $z a$ is always in $A$ for any $z$ in $O_{K}$, then $A$ is called a fractional ideal. For a fractional ideal $A$, there always exists (not unique) some rational integer $m$ such that $m A$ is in $O_{K}$, i. e., $m A$ is an integral ideal.

For two (integral or fractional) ideals $A$ and $B$, if there exists an integral ideal $C$ such that $A=B C$, we denote $B \mid A$. Otherwise we denote $B \nmid A$. A useful fact is that $B \mid A$ iff $A \subset B$.

With the same embeddings specified in (3) and the bilinear form in (3.5) associated with a fractional ideal $A, A$ also becomes a lattice in $R^{n}$, so all the lattice problems, e.g., SVP and CVP, are meaningful to fractional ideals.

Note: Hereafter we interchangeably use the terminology "ideal" and "ideal lattice" .

With the viewpoint of extension from $Q$ to $K$, one of the most subtle phenomena is when and how a prime number $p$ (irreducible in $Z$ ) becomes reducible in the extended number field $K$. Such phenomena is used as a critical tool in our approach and we briefly present the facts about it in next section in a more general viewpoint. 


\subsection{More General Model: Relative Extension and Prime Ideal Decomposition}

The theory sketched in Sect.3.2 can be deepen to notions and principles in a more general model. Let $K$ be a number field with its integral ring $O_{K}$ (or more generally, a fractional field of a Dedekind domain $\left.O_{K}[16,17]\right), L=K(\alpha)$ is an extension of $K$ by adding a root $\alpha$ of a polynomial $f(x) \in O_{K}[x] . f(x)$ is called $\alpha$ 's minimal polynomial if it has the minimal degree among the polynomials in $O_{K}[x]$ with $\alpha$ as a root. Such a polynomial is unique up to a constant factor in $O_{K}$ and is prime (irreducible) in $O_{K}[x]$. The minimal polynomial $f(x)$ 's degree is called the degree of field extension $L / K$ and denoted by $[L: K]$.

$L / K$ is called relative extension from the ground field $K$. The theory in Sect.3.2 is only about the case where the ground field is $Q$. As in the case of $K / Q$, an equivalent (isomorphic) picture about the arithmetic in $L$ is to regard it as the quotient set of $O_{K}[x] /(f(x))$ with the operations as polynomial addition, subtraction and multiplication modulo $f(x)$.

Regarding $L$ as a vector space on $K$ with dimension $n=[L: K]$, we can introduce the relative trace and norm for any element $z$ in $L$ as what is done in 3.2 and denote these as $\operatorname{Tr}_{L / K}(z)$ and $N_{L / K}(z)$ respectively[14-16]. For any relative extension $L / M / K$, an important fact is that:

$$
\begin{aligned}
\operatorname{Tr}_{L / K}(z) & =\operatorname{Tr}_{M / K}\left(\operatorname{Tr}_{L / M}(z)\right) \\
N_{L / K}(z) & =N_{M / K}\left(N_{L / M}(z)\right)
\end{aligned}
$$

for any $z$ in $L$.

Given $z$ 's minimal polynomial $g(t)=(-1)^{m} g_{0}+g_{1} z+\ldots+g_{m-1} t^{m-1}+$ $t^{m}$ in $K[t]$ (hence $\left.m \mid n\right)$, $z$ 's trace and norm can be computed by

$$
\operatorname{Tr}_{L / K}(z)=-(n / m) g_{m-1}, N_{L / K}(z)=g_{0}^{n / m}
$$

For relative extension $L / K$, there is an important subset, called $O_{K}$ 's integral closure in $L$, defined as:

$O_{L} \equiv\left\{z\right.$ in $L:$ there exist $a_{0}, \ldots, a_{n-1}$ in $O_{K}$ such that $\left.a_{0}+a_{1} z+\ldots+a_{n-1} z^{n-1}+z^{n}=0\right\}$

$O_{L}$ is a ring with the following important properties[11, 14-16]:

(1) $L$ is $O_{L}$ 's fractional field.

(2)For an (integral) ideal $A$ in $O_{L}$ (including $O_{L}$ itself), there may not exist any set of $O_{K}$-linear independent elements $\omega_{1}, \ldots, \omega_{n}$ in $A$ where $n=[K: Q]$ such that every element in $A$ can be uniquely represented as a $O_{K}$-coefficient linear combination on $\omega_{1}, \ldots, \omega_{n}$, unless when $O_{K}$ is the so-called principal ideal ring.

(3)For any relative extension $L / K$ of degree $n$, there are exactly $n$ field (relative) embeddings (injective homomorphisms) mapping $L$ into $C$ which are fixed in $K$ element-wise, among which $\rho_{1}, \ldots, \rho_{r_{1}}$ embed $L$ into $R$ and the other $2 r_{2}$ ones $\tau_{1}, \ldots, \tau_{2 r_{2}}$ (where each $\tau_{j}$ is complex conjugate to $\tau_{j+r_{2}}$ ) embed $L$ into C.

With these $n K$-embeddings $\sigma_{1}, \ldots, \sigma_{n}$, the trace and norm of an element can be computed by

$$
\operatorname{Tr}_{L / K}(z)=\sigma_{1}(z)+\ldots+\sigma_{n}(z), N_{L / K}(z)=\sigma_{1}(z) \ldots \sigma_{n}(z)
$$

As long as $K$ is a number field, $L$ is also a number field with degree $[L: Q]=$ $[L: K][K: Q]$ and $O_{L}$ defined in (3.8) is exactly the set of $\{z$ in $L$ : there 
exist $a_{0}, \ldots, a_{m-1}$ in $Z$ such that $\left.a_{0}+a_{1} z+\ldots+a_{m-1} z^{m-1}+z^{m}=0\right\}$ where $m=[L: Q]$. Therefore any ideal $\mathrm{A}$ in $O_{L}$ can be regarded, by the number field $L$ 's embeddings into $C$, as a lattice of dimension $[L: Q]$ in $R^{[L: Q]}$ with the positive-definite and non-degenerate bilinear form

$$
<x, y>\equiv \operatorname{Tr}_{L / Q}(x \bar{y}) \text { where } \bar{y} \text { denotes } y^{\prime} \text { s complex conjugation }
$$

Because $L$ is a number field, as a result, every prime ideal $M$ in $O_{L}$ is maximal and $O_{L} / M$ is a (finite) field. The important property of the unique factorization on prime ideals is certainly also true for any ideal in $O_{L}$.

(4)Let $P$ be a prime ideal in $O_{L}$, generally the ideal $P O_{L}$ may be no longer prime in $O_{L}$. As an ideal in $O_{L}$, there is the following law about $P O_{L}$ 's decomposition:

For any prime ideal $P$ in $O_{K}$, there exist a finite set of prime ideals $M_{1}, \ldots, M_{r}$ in $O_{L}$ such that $M_{1} \cap O_{K}=\ldots=M_{r} \cap O_{K}=P$ and $P O_{L}$ decomposes into prime ideals multiplication on and only on these $M_{1}, \ldots, M_{r}$ :

$$
P O_{L}=M_{1}^{e_{1}} \ldots M_{r}^{e_{r}}
$$

Furthermore, $e_{1} f_{1}+\ldots+e_{r} f_{r}=[L: K]$ where $f_{i}=\left[O_{L} / M_{i}: O_{K} / P\right]=$ the degree of the extension from the finite field $O_{K} / P$ to $O_{L} / M_{i}$. Integers $e_{1}, \ldots, e_{r}$ are called ramification indices for $P$ on $M_{1}, \ldots, M_{r}$ (or $M_{1}, \ldots, M_{r}$ on $P$ ).

Remarks on Galois Extension: When $L / K$ is a Galois extension, the decomposition law (3.11) can be further refined. In this case we always have $e_{1}=\ldots=e_{r} \equiv e$ and $f_{1}=\ldots=f_{r} \equiv f$. Furthermore, Galois group $G_{L / K}$ is transitive on $M_{1}, \ldots, M_{r}$, i.e., for any $M_{i}, M_{j}$ there exists $g$ in $G_{L / K}$ such that $M_{i}=g\left(M_{j}\right)$.

\subsection{Valuations, p-adic Completions and Local-Global Relations}

Section 3.2-3.3 presented number theory on the so-called global field. Now we turn to number theory on the so-called local field.

General Notions and Facts Let $K$ be a field, $R^{+}$be the set of all nonnegative real numbers, a (multiplicative) valuation on $K$ is a mapping $||:. K \rightarrow$ $R^{+}$with the following properties:

$$
|x y|=|x||y| ;|x|=0 \text { iff } x=0 ;|x+y| \leq|x|+|y| \text { for any } x \text { and } y \text { in } K
$$

When $|n| \leq 1$ for all $n=0, \pm 1, \pm 2, \pm 3, \ldots,|$.$| is called non-Archimedean valu-$ ation, otherwise called Archimedean. For non-Archimedean valuation, the third property in the above is equivalent to the inequality

$$
\begin{aligned}
|x+y| & \leq \max (|x|,|y|) \text { for any } x \text { and } y \text { in } K \\
\text { Or equivalently }|x+y| & =\max (|x|,|y|) \text { for any } x \text { and } y \text { in } K \text { and }|x| \neq|y|
\end{aligned}
$$

An equivalent non-Archimedean valuation model is the index valuation, i. e., a mapping $w:||:. K \rightarrow R$ satisfying $w(x y)=w(x)+w(y) ; w(x)=+\infty$ iff $x=$ $0 ; w(x+y) \geq \min (w(x), w(y))$ for any $x$ and $y$ in $K$. Obviously, for any $a>$ $1 w(x)=-\log _{a}|x|$ gives the correspondance between these two models.

Note: Hereafter we freely interchange the use of these two valuation models at convenience. 
Two (multiplicative) valuations $|\cdot|_{1}$ and $|\cdot|_{2}$ on filed $K$ is called equivalent if there exists a positive real number $a>0$ such that $|x|_{1}=|x|_{2}^{a}$ for all $x$ in $K$. For two non-Archimedean valuations $|\cdot|_{1}$ and $|\cdot|_{2}$, this definition equals the statement that $|x|_{1} \leq 1$ iff $|x|_{2} \leq 1$ for any $x$ in $K$.

A valuation |.| is called discrete if the image of $|$.$| is discrete in R$.

Given a non-Archimedean valuation |.|(or its equivalent index valuation $w$ ) on field $K$, the subset

$$
J_{K} \equiv\{x \text { in } K:|x| \leq 1\}=\{x \text { in } K: w(x) \geq 0\}
$$

is a ring with the unique maximal ideal[13, 16]:

$$
M_{K} \equiv\{x \text { in } K:|x|<1\}=\{x \text { in } K: w(x)>0\}
$$

The field $J_{K} / M_{K}$ is called the valuation's residue class field.

For number field $K / Q$ with degree $n=[K: Q]$ we have the following important general facts about valuations on it[13]:

(1)Each (real or complex) $Q$-embedding $\sigma_{j}: K \rightarrow C$ (r.f. Sect.3.2(3)) derives an Archimedean (multiplicative) valuation on $K$ by $|x|_{j} \equiv\left|\sigma_{j}(x)\right|$ where the latter $|$.$| is the ordinary complex valuation |z|=\left((\operatorname{Re} z)^{2}+(\operatorname{Im} z)^{2}\right)^{1 / 2}$. Furthermore, two derived Archimedean valuations $|\cdot|_{j}$ and $|.|_{i}$ are equivalent iff $\sigma_{j}($. and $\sigma_{i}($.$) are complex conjugate each other.$

(2)Each prime ideal $P$ in $O_{K}$ derives a discrete non-Archimedean (index) valuation on $K$ by

$$
w_{P}(x) \equiv \text { e where } P^{e} \mid(x) \text { and } P^{e+1} \nmid(x)(r . f . \text { notations in Sect.3.2(7)) }
$$

This is called the $P$-adic valuation. Furthermore, different prime ideals $P_{i}, P_{j}$ derive distinct (inequivalent) $P$-adic valuations $w_{P_{i}}, w_{P_{j}}$.

(3)The valuations presented in (1) and (2) enumerates all valuations on the number field $K$. As a result, there are finite (exactly $r_{1}+r_{2}$ ) number of Archimedean valuations and infinite distinct non-Archimedean valuations, each corresponding to a prime ideal.

Example On rational number field $Q$, the only Archimedean valuation |.| is the ordinary absolute value and each non-Archimedean valuation is corresponding to a prime number $p$, i. e., the $p$-adic valuation. For example, with the standard $p$-valuation we have, for any integer $m$ :

$$
|m|_{p}=p^{-e} \text { if } p^{e} \mid m \text { but } p^{e+1} \nmid m
$$

In particular, $|m|_{p}=1$ iff $m$ and $p$ are co-prime.

Completeness and Local Field Let $K$ be a field with a (Archimedean or nonArchimedean) valuation |.|. Since |.| derives a metric on $K$ by $d(x, y) \equiv|x-y|$, the standard metric-completion procedure derives a |.|-completion on $K$, denoted $K_{\|}$, which is also a field with $K$ as a dense subfield in it.

Let $K$ be a number field. The completion by anyone of its Archimedean valuations is $R$ or $C$, depending on whether $K$ is a subfield in $R$ or not. Let $P$ be a prime ideal in $O_{K}$, the $P$-adic completion of $K$, denoted $K_{P}$ and called $K$ 's localization on $P$ (local field), has the following properties[13, 16]:

(1) $K_{P}$ is a complete and discrete valued field. Further more, $K_{P} / Q_{p}$ is a finite-degree extension where $p$ is a prime number such that $(p) \equiv p Z=P \cap Z$ and $Q_{p}$ is the $p$-adic completion of the field of rational numbers $Q$. 
(2)For $K_{P}$ 's valuation ring (r.f., (3.13a)) we have

$$
\begin{aligned}
J_{K, P} \equiv & \left\{x \text { in } K_{P}:|x|_{P} \leq 1\right\} \equiv\left\{x \text { in } K_{P}: w_{P}(x) \geq 0\right\} \\
= & \left\{x \text { in } K_{P}: \text { there exist } a_{0}, \ldots, a_{n-1} \text { in } Q_{p} \text { such that } w_{p}\left(a_{i}\right) \geq 0 \text { for all } i\right. \\
& \text { and } \left.a_{0}+a_{1} x+\ldots+a_{n-1} x^{n-1}+x^{n}=0\right\}
\end{aligned}
$$

Furthermore $J_{K, P}$ is a principal ideal domain with the unique maximal ideal:

$$
M_{K, P}=\left\{x \text { in } K_{P}:|x|_{P}<1\right\}=\left\{x \text { in } K_{P}: w_{P}(x)>0\right\}
$$

Hence there exists a element $\pi$, called $K_{P}$ 's prime element, such that $M_{K, P}=$ $(\pi)$. Actually $\pi$ can be any element in $M_{K, P}$ with the greatest $\left.|\cdot|\right|_{P}$-value.

(3) Given an ideal $B$ in $J_{K, P}$, there exists a unique integer $m \geq 0$ such that $B=M_{K, P}^{m}$. In consequence, all integral ideals in $J_{K, P}$ constitute a chain $\ldots \subset M_{K, P}^{4} \subset M_{K, P}^{3} \subset M_{K, P}^{2} \subset M_{K, P}$.

(4)The residue class field of $K_{P}$, i. e., $J_{K, P} / M_{K, P}$, is a finite field with characteristic $p$ (the $p$ specified in (1)) and isomorphic to $O_{K} / P$.

(5)There is a homomorphism $\Omega$ mapping the ideals in $O_{K}$ to ideals in $J_{K, P}$, defined as:

$$
\Omega(A)=M_{K, P}^{e} \text {, if } P^{e} \mid A \text { but } P^{e+1} \nmid A ; \Omega(A)=J_{K, P} \text { if } P \text { and } A \text { are co-prime }
$$

It's easy to verify that $\Omega(A B)=\Omega(A) \Omega(B)$ and $\Omega$ can be easily prolonged onto the multiplicative group of fractional ideals on $K$. $\Omega$ "localizes" an ideal $A$ in global field $K$ to a (principal) ideal $\Omega(A)$ in $K_{P}$ and this localization is non-trivial iff $P$ is a prime factor of $A$.

Local-Global Relations Now back to Sect.3.3(4), let both $L$ and $K$ be number fields and $L / K$ a field extension of degree $n=[L: K], P$ a prime ideal in $O_{K}$. There exist a finite set of prime ideals $M_{1}, \ldots, M_{r}$ in $O_{L}$ and integers $e_{1}, \ldots, e_{r} \geq 1$ such that :

$$
\begin{gathered}
M_{1} \cap O_{K}=\ldots=M_{r} \cap O_{K}=P \\
P O_{L}=M_{1}^{e_{1}} \ldots M_{r}^{e_{r}} \\
e_{1} f_{1}+\ldots+e_{r} f_{r}=n
\end{gathered}
$$

where $f_{i}=\left[O_{L} / M_{i}: O_{K} / P\right]=$ the degree of the extension from the finite field $O_{K} / P$ to $O_{L} / M_{i}$.

Let $L_{M_{j}}$ be the $M_{j}$-adic completion of $L$ with its valuation ring denoted by $J_{M_{j}}$, prime element $\eta_{j}$ (i. e., $\left.J_{M_{j}}=\left(\eta_{j}\right)\right), j=1, \ldots, r, K_{P}$ be the $P$-adic completion of $K$ with its valuation ring denoted by $J_{K, P}$ and prime element $\pi$, now we can state more important and deep details about this decomposition $\operatorname{law}[13]$ :

(1)Each $L_{M_{j}}$ is an extension of $K_{P}$ and $\left[L_{M_{j}}: K_{P}\right]=e_{j} f_{j}, j=1, \ldots, r$. In particular, each $L_{M_{j}}$ is a vector space on local field $K_{P}$ in dimension $e_{j} f_{j}$.

(2)For each $j$, the residue class field of $L_{M_{j}}$ is an extension of the residue class field of $K_{P}$ with degree $f_{j}$, i. e., $\left[J_{M_{j}} /\left(\eta_{j}\right): J_{K, P} /(\pi)\right]=f_{j}$.

(3)For each $j$, the ground field prime element is decomposed in the extended local field with ramification index $e_{j}$, i. e., $(\pi)=\left(\eta_{j}\right)^{e_{j}}$ in $J_{M_{j}}$. 
(4)For each $j$, there is a prolongation from the $P$-adic valuation on $K_{P}$ to $L_{M_{j}}$ specified by

$$
|y|_{M_{j}}=\left|N_{L_{M j} / K_{P}}(y)\right|_{P}^{1 / e_{j} f_{j}} \text { for any } y \text { in } L_{M_{j}} .
$$

where $|\cdot|_{P}$ denotes the $P$-adic multiplicative valuation on $K_{P}$. It's easy to see that $|y|_{M_{j}}=|y|_{P}$ when $y$ is in $K_{P}$. Furthermore, $|y|_{M_{j}}$ in (3.17) is the only prolongation of $|\cdot|_{P}$ onto $L_{M_{j}}$.

(5)For each $j, L_{M_{j}}$ 's valuation ring $J_{M_{j}}$ is exactly the integral closure of $K_{P}$ 's valuation $\operatorname{ring} J_{K, P}$, i. e.,

$$
\begin{aligned}
& J_{M_{j}} \equiv\left\{y \text { in } L_{M_{j}}:|y|_{M_{j}} \leq 1\right\} \equiv\left\{y \text { in } L_{M_{j}}: w_{M_{j}}(y) \geq 0\right\} \\
& \quad=\left\{y \text { in } L_{M_{j}}: \text { there exist } a_{0}, \ldots, a_{m-1} \text { such that } w_{P}\left(a_{i}\right) \geq 0\left(i . e ., \text { in } J_{K, P}\right)\right. \\
& \left.\quad \text { for all } i \text { and } a_{0}+a_{1} x++a_{m-1} y^{m-1}+y^{m}=0\right\}
\end{aligned}
$$

(6)Let $L=K \omega_{1}+\ldots+K \omega_{n}$ and w.l.o.g., all $\omega_{i}$ 's are in $O_{K}$. Denote the vector space $K_{P} \omega_{1}+\ldots+K_{P} \omega_{n}$ on field $K_{P}$ by $K_{P} \otimes_{K} L$ (tensor product on $K)$ and denote the direct summation between vector spaces by $\oplus$, there is a $K_{P}$-linear isomorphism $\psi$ between $K_{P} \otimes L$ and $L_{M_{1}} \oplus \ldots \oplus L_{M_{r}}$ where each $L_{M_{i}}$ is a (distinct) vector space on $K_{P}$ in dimension $e_{j} f_{j}$ :

$$
\psi: K_{P} \otimes_{K} L \cong L_{M_{1}} \oplus \ldots \oplus L_{M_{r}}
$$

Furthermore, denote the element corresponding in $(3.19)$ as $y \cong\left(y_{1}, \ldots, y_{r}\right)$ then for any $y$ in $L$ we have

$$
\begin{gathered}
\operatorname{Tr}_{L / K}(y)=\operatorname{Tr}_{L_{M_{1} / K_{P}}}\left(y_{1}\right)+\ldots+\operatorname{Tr}_{L_{M_{r}} / K_{P}}\left(y_{r}\right) \\
N_{L / K}(y)=N_{L_{M_{1}} / K_{P}}\left(y_{1}\right) \ldots N_{L_{M_{r}} / K_{P}}\left(y_{r}\right)
\end{gathered}
$$

Let $y^{(1)} \cong\left(y_{1}^{(1)}, \ldots, y_{r}^{(1)}\right)$ and $y^{(2)} \cong\left(y_{1}^{(2)}, \ldots, y_{r}^{(2)}\right)$, at element level the isomorphism has:

$$
\begin{gathered}
y^{(1)} \pm y^{(2)} \cong\left(y_{1}^{(1)} \pm y_{1}^{(2)}, \ldots, y_{r}^{(1)} \pm y_{r}^{(2)}\right) \\
y^{(1)} y^{(2)} \cong\left(y_{1}^{(1)} y_{1}^{(2)}, \ldots, y_{r}^{(1)} y_{r}^{(2)}\right)
\end{gathered}
$$

Combined with (3.20a) and (3.21a) we have

$$
\operatorname{Tr}_{L / K}(x y)=\operatorname{Tr}_{L_{M_{1}} / K_{P}}\left(x_{1} y_{1}\right)+\ldots+\operatorname{Tr}_{L_{M_{r}} / K_{P}}\left(x_{r} y_{r}\right)
$$

for $L$ 's any element $x \cong\left(x_{1}, \ldots, x_{r}\right)$ and $y \cong\left(y_{1}, \ldots, y_{r}\right)$. In other words, $(3.19)$ presents an orthogonal decomposition of the $K_{P}$-vector space $K_{P} \otimes_{K} L$.

(7)Let $A$ be any (integral or fractional) ideal in $L$, then $A$ is a finitely generated module on the Dedekind domain. There exist $L$ 's $K$-basis $\omega_{1}, \ldots, \omega_{n}$ and a set of $K$ 's ideals $I_{1}, \ldots, I_{n}$ such that $[17,20]$

$$
A=I_{1} \omega_{1}+\ldots+I_{n} \omega_{n}
$$

Such $\omega_{1}, \ldots, \omega_{n}$ are called $A$ 's pseudo-basis and in general they are not in $A$. Different pseudo-basis share the same cardinality $n$ and it is known how to transform from one pseudo-basis to another[20]. 
Let $A$ has a pseudo-basis representation in (3.23), define $J_{K, P} \otimes_{K} A \equiv$ $I_{P}^{(1)} \omega_{1}+\ldots+I_{P}^{(n)} \omega_{n}$ where $I_{P}^{(i)}=I_{i}$ 's image under the localization mapping $\Omega$ in (3.15) in $K$. Let $\Omega_{j}$ be the localization mapping in (3.15) in $L_{M_{j}}$, i. e., mapping the ideals in $L$ to ideals in $L_{M_{j}}$, then we have the following fact.

Theorem 3.1. [21] If A's pseudo-basis $\omega_{1}, \ldots, \omega_{n}$ are in $O_{L}$, then the $K_{P^{-}}$ linear isomorphism $\psi$ in (3.19) deduces:

$$
\psi: J_{K, P} \otimes_{K} A \cong \Omega_{1}(A) \oplus \ldots \oplus \Omega_{r}(A)
$$

\section{Local-Global Algorithm to Solve SVP and CVP in Ideal Lattices: High Level Descriptions}

In this section we construct our algorithms to solve SVP and CVP in ideal lattices. Only the search version is considered because the optimization version can be solved in exactly the same way. Furthermore, we only focus on SVP because the same approach can be easily applied to CVP.

\subsection{Problem}

The search shortest vector problem in ideal lattice is presented in the following. Instead of only dealing with the case $K / Q$, our algorithm works for any finitedegree relative extension $L / K$ where $K$ is fixed and $L$ is arbitrary, both are number fields.

\section{- Problem SVP $(A, L / K)$}

Parameter: $A$ number field $K$.

Input: $K$ 's extended field $L=K(\alpha)$ with the generator $\alpha$ 's minimal polynomial $f(t)=t^{n}+a_{1} t^{n-1}+\ldots+a_{n-1} t+a_{0}$ in $O_{K}[t]$, and an ideal $A$ in $L$.

Note: In this paper we only deal with the case of real number field, i.e., $L$ is contained in $R$.

For the ideal $A$ on input, we always assume a given pseudo-basis representation, i. e., a set of $L$ 's $K$-basis $\omega_{1}, \ldots, \omega_{n}$ in $O_{L}$ and a set of $K$ 's ideals $I_{1}, \ldots, I_{n}$ such that $A=I_{1} \omega_{1}+\ldots+I_{n} \omega_{n}$.

Output: An element $y^{*}$ in $A$ such that

$$
\operatorname{Tr}_{L / K}\left(y^{* 2}\right)=\min \left\{\operatorname{Tr}_{L / K}\left(y^{2}\right): \text { all non-zero } y \text { 's in } \mathrm{A}\right\}
$$

\subsection{High Level Algorithm}

Before going to the technically involved solver construction, we briefly present the motivation. The idea comes from a simple fact that, although lattice problems (e.g., SVP, CVP, CRP, etc) are computationally hard in general cases, a subset of them, in particular the problems of the orthogonal lattice family, can be always solved with polynomial-complexity algorithms. Of course for a general lattice in $R^{n}$ neither it is always orthogonal nor it can be even decomposed to a set of sub-lattices orthogonal each other, however, for ideal lattices originating 
from number field, (3.19)-(3.24) shows that there exists some "orthogonal decomposition" structure exploitable to develop a solver more efficient than those of general lattice problems. Doing such exploitations as far as possible is exactly what will proceed in this paper.

Now we present the whole algorithm's logic at a high level, then working out all technical details in sequel. In the following, all notations are inherited from Sect.3 and "s.t." means "such that". By "global field" we mean any number field and "local field" means the completion of a number field under some of its prime ideal induced valuation.

\section{Algorithm for $\operatorname{SVP}(\mathbf{A}, L / K)$ : High-Level}

(1) Given $L$ and $A$ on input, find a prime ideal $P$ in $O_{K}$ such that:

$$
P O_{L} \text { is not prime in } O_{L} \text {; }
$$

$$
P \text { is unramified in } O_{L} \text {, i.e., }
$$

all its ramification indices $e_{1}=\ldots=e_{r}=1$;

$$
P \nmid \sharp\left(O_{L} / O_{K}[\alpha]\right) .
$$

(2)Given $L$ and $P$ obtained from last step, find the local fields $L_{M_{1}}, \ldots, L_{M_{r}}$ associated with $P$ 's all decomposition prime ideals $M_{1}, \ldots, M_{r}$ in $O_{L}$, integers $f_{1}, \ldots, f_{r} \geq 1$ s.t. :

$$
P O_{L}=M_{1} \ldots M_{r}
$$

$f_{i}=\left[O_{L} / M_{i}: O_{K} / P\right]=\left[L_{M_{i}}\right.$ 's residue class field : $K_{P}$ 's residue class field $]$

Secondly, find $K_{P}$-linear isomorphism $\psi$ and its component mappings $\psi_{1}, \ldots, \psi_{r}$ in (3.19)-(3.21) where each $\psi_{i}: L \rightarrow L_{M_{i}}$, i. e., $y \cong\left(y_{1}, \ldots, y_{r}\right)$ means $\psi(y)=\left(\psi_{1}(y), \ldots, \psi_{r}(y)\right)$.

(3) Given $L, P$ and $L_{M_{1}}, \ldots, L_{M_{r}}$, integers $f_{1}, \ldots, f_{r} \geq 1$ obtained from last step, find $K$ 's extended fields $L_{1}, \ldots, L_{r}$ and field embeddings $\varphi_{1}, \ldots, \varphi_{r}$ with $\varphi_{i}: \psi_{i}(L) \rightarrow L_{i}$, s.t.:

Each $L_{i}$ is a global field with extension degree $\left[L_{i}: K\right]=f_{i}$;

For each $i$ and $y$ in $L: \operatorname{Tr}_{L_{i} / K}\left(\varphi_{i} \psi_{i}(y)\right)=\operatorname{Tr}_{L_{M_{i} / K_{P}}}\left(\psi_{i}(y)\right) ; \quad(4.2 \mathrm{~b})$ (4)Given all the results obtained, for each $i$ set $\lambda_{i} \equiv \varphi_{i} \psi_{i}: L \rightarrow L_{i}$ and $A_{i} \equiv \lambda_{i}(A)$ which is an ideal in $L_{i}$. Do:

For each $i=1, \ldots, r$ find a non-zero $x_{i}^{*}$ in $A_{i}$ s.t.

$$
\operatorname{Tr}_{L_{i} / K}\left(x_{i}^{* 2}\right)=\min \left\{\operatorname{Tr}_{L_{i} / K}\left(x^{2}\right): \text { all non-zero } x^{\prime} \mathrm{s} \text { in } A_{i}\right\}
$$

ie, solve the SVP for ideal lattice $A_{i}$ in field $L_{i}$ in a strictly lower dimension $f_{i}(<n)$;

Find a $x_{m}^{*}$ among $x_{1}^{*}, \ldots, x_{r}^{*}$ s.t.

$$
\operatorname{Tr}_{L_{m} / K}\left(x_{m}^{* 2}\right)=\min \left\{\operatorname{Tr}_{L_{i} / K}\left(x_{i}^{* 2}\right): i=1, \ldots, r\right\} ;
$$

Find $y^{*}$ in $A$ such that

$$
\lambda_{m}\left(y^{*}\right)=x_{m}^{*} \text { and } \lambda_{i}\left(y^{*}\right)=0 \text { for all } i \neq m ;
$$

$\operatorname{Output}\left(y^{*}\right)$ 
Such obtained $y^{*}$ is indeed the solution because

$$
\begin{aligned}
& \min \left\{\operatorname{Tr}_{L / K}\left(y^{2}\right): \text { all non-zero } y \text { 's in } A\right\} \\
= & \min \left\{\operatorname{Tr}_{L_{M_{1}} / K_{P}}\left(y_{1}^{2}\right)+\ldots+\operatorname{Tr}_{L_{M_{r}} / K_{P}}\left(y_{r}^{2}\right): \text { any } y_{i} \text { in } \psi_{i}(A)\right. \\
& \text { and } \left.y_{i}=0 \text { doesn't hold simultaneously }\right\} \text { by }(3.20),(3.21) \operatorname{and}(3.24) \\
= & \min \left\{\operatorname{Tr}_{L_{1} / K}\left(x_{1}^{2}\right)+\ldots+\operatorname{Tr}_{L_{r} / K}\left(x_{r}^{2}\right): \text { any } x_{i} \text { in } A_{i}\right. \\
& \left.\operatorname{and} x_{i}=0 \text { doesn't hold simultaneously }\right\} \text { by }(4.2) \\
= & \min _{1 \leq i \leq r} \min \left\{\operatorname{Tr}_{L_{i} / K}\left(x_{i}^{2}\right): \text { any } x_{i} \text { in } A_{i} \text { and non-zero }\right\} \\
= & \min _{1 \leq i \leq r} \operatorname{Tr}_{L_{i} / K}\left(x_{i}^{* 2}\right)
\end{aligned}
$$

Note that in step $\sharp 2$ we don’t need $P$ 's decomposition prime ideals per se, but just some information about their local fields $L_{M_{1}}, \ldots, L_{M_{r}}$ where each $L_{M_{i}}=K_{P}[t] /\left(f_{i}(t)\right)$ with some irreducible polynomial $f_{i}(t)$ in $K_{P}[t]$. In the low-level constructions it can be seen that even $f_{i}(t)$ is not needed but just the polynomial $h_{i}(t)=f_{i}(t) \bmod P$ in $K[t]$ instead

In conclusion, this algorithm reduces an ideal lattice SVP instance of dimension $n$ to a set of $r(\leq n)$ ideal lattice SVP instances of strictly lower dimensions. It's already known that lattice problem's computational hardness is only dominated by its dimension $n$ (in other words, there are known algorithms in polynomial time and space complexity to solve lattice problems like SVP and CVP for any fixed dimension $[3-5,7-9,22]$ ), this feature of our algorithm is significantly helpful to raise the solver's efficiency in solving ideal lattice SVP.

For all those derived sub-instances the ground field are all $K$, the same as that in the original SVP instance, so (4.3) in step $\sharp 4$ can be recursively solved by this algorithm down to some appropriately lower dimensions, calling some existed solver at these levels or continue the recursion down to 1-dimensional SVP sub-instances.

\section{Remarks:}

(1)For CVP of ideal lattices, i. e., on input the extended (real) field $L=K(\alpha)$, ideal $A$ in $L$ and an element $z$ in $L$, to find $y^{*}$ in $A$ s.t.

$$
\operatorname{Tr}_{L / K}\left(\left(z-y^{*}\right)^{2}\right)=\min \left\{\operatorname{Tr}_{L / K}\left((z-y)^{2}\right): \text { all } y^{\prime} s \text { in } A\right\}
$$

Because:

$$
\begin{aligned}
& \min \left\{\operatorname{Tr}_{L / K}\left((z-y)^{2}\right): \text { all non-zero y's in } A\right\} \\
= & \min \left\{\operatorname{Tr}_{L_{M_{1} / K_{P}}\left(\left(z-y_{1}\right)^{2}\right)+\ldots+\operatorname{Tr}_{L_{M_{r}} / K_{P}}\left(\left(z-y_{r}\right)^{2}\right):}\right. \\
& \text { any } \left.y_{i} \text { in } \psi_{i}(A)\right\} \text { by }(3.20),(3.21),(3.24) \\
= & \min \left\{\operatorname{Tr}_{L_{1} / K}\left(\left(\lambda_{1}(z)-x_{1}\right)^{2}\right)+\ldots+\operatorname{Tr}_{L_{r} / K}\left(\left(\lambda_{r}(z)-x_{r}\right)^{2}\right):\right. \\
& \left.\operatorname{any} x_{i} \text { in } A_{i}\right\} \text { by }(4.2) \\
= & \min _{1 \leq i \leq r} \min \left\{\operatorname{Tr}_{L_{i} / K}\left(\left(\lambda_{i}(z)-x_{i}\right)^{2}\right): \text { any } x_{i} \text { in } A_{i}\right\} \\
= & \min _{1 \leq i \leq r} \operatorname{Tr}_{L_{i} / K}\left(\left(\lambda_{i}(z)-x_{i}^{*}\right)^{2}\right)
\end{aligned}
$$

solving the CVP of ideal lattices can be done by a similar algorithm following the logics of that for SVP. For this reason, we will only focus on solving SVP 
hereafter.

(2)In the first step, if such a prime ideal $P$ is found that completely splits in the extended field $L$, i. e., $P O_{L}=M_{1} \ldots M_{n}$, then solving the SVP instance in this case is reduced to solving $n$ 1-dimensional SVP instances of some ideals in $K$. (3)In case of Galois extension $L / K$, the decomposition law (4.1) will have $e_{1}=$ $\ldots=e_{r} \equiv e=1$ and $f_{1}=\ldots=f_{r} \equiv f$. Since $r e f=n$ and $r \geq 2$, we always have $f=n / r \leq n / 2$, i. e., each reduction can decrease the instance's dimension by at least half and at most $O(\log n)$ recursions are needed.

For example, supposing that each recursion reduces the dimensions (the intermediate fields' extension degrees on $\mathrm{K}$ ) by half, then after $m$ recursions the original $n$-dimensional SVP instance will be decomposed to $2^{m}$ number of $n / 2^{m}$ dimensional SVP instances. As a result, the time complexity would be at most $2^{m} 2^{O\left(n / 2^{m}\right)}$ by calling some single-exponential time-complexity generic solvers on these $n / 2^{m}$-dimensional SVP instances(e.g., the elegant solver in [6]), substantially more efficient than the time-complexity of $2^{O(n)}$ if the $n$-dimensional original instance is directly solved.

(4)In the case of Galois extension $L / K$, the fact that Galois group $G_{L / K}$ is transitive on $M_{1}, \ldots, M_{r}$ in (4.1), i. e., for any $M_{i}, M_{j}$ there exists $g$ in $G_{L / K}$ such that $M_{i}=g\left(M_{j}\right)$, can significantly simplify lots of details in our algorithm's construction.

(5)In general cases ( $L / K$ may not be Galois), to make the sub-instances' dimensions as low as possible at each recursion, it's helpful to find a prime ideal $P$ in $O_{K}$ not only satisfying those requirements in step $\sharp 1$ but also the objective that $\max _{j=1, \ldots, r} f_{j}$ is as small as possible, where $r, f_{1}, \ldots, f_{r}$ are those integers appearing in step $\sharp 2$.

\section{Low Level Details in the Algorithm}

Now we turn from the high-level descriptions to low-level technical details, each step discussed in a subsection. We begin with the relatively easy step $\sharp 2, \sharp 3$ and $\sharp 4$ and finally end with step $\sharp 1$.

\subsection{Solving Subproblems in Step $\sharp 2$}

In this step we solve such problems: Given $L=K(\alpha)$ with $\alpha$ 's minimal monic polynomial $f(t) \in K[t]$ and unramified prime ideal $P$ in $O_{K}$ where $P \nmid$ $\sharp\left(O_{L} / O_{K}[\alpha]\right)$, firstly, find irreducible polynomials $h_{1}(t), \ldots, h_{r}(t) \in K[t]$ s.t. $h_{i}(t)=f_{i}(t) \bmod P$ where $L_{M_{i}}=K_{P}[t] /\left(f_{i}(t)\right)$ 's are local fields associated with $P$ 's all decomposition prime ideals $M_{1}, \ldots, M_{r} \subset O_{L}$. Note that in this situation naturally (i. e.,due to $P$ 's unramification) each $\operatorname{degh} h_{i}(t)=\operatorname{deg} f_{i}(t)=\left[O_{L} / M_{i}\right.$ : $\left.O_{K} / P\right]=\left[L_{M_{i}}\right.$ 's residue class field : $K_{P}$ 's residue class field $]$. Secondly, find $K_{P^{-}}$ linear isomorphism $\psi$ and its component mappings $\psi_{1}, \ldots, \psi_{r}$ in (3.19)-(3.21) where each $\psi_{i}: L \rightarrow L_{M_{i}}$, i. e., $y \cong\left(y_{1}, \ldots, y_{r}\right)$ means $\psi(y)=\left(\psi_{1}(y), \ldots, \psi_{r}(y)\right)$.

\section{Solution to the 1st sub-problem:}

Decompose $f(t) \bmod P$ by calling any appropriate polynomial factorization algorithm modulo the prime ideal(e. g., those in $[20,23,24])$,i.e., to compute distinct monic irreducible polynomials $h_{1}(t), \ldots, h_{r}(t) \in$ $\left(O_{K} / P\right)[t]$ (hence irreducible in $\left.K[t]\right)$ s.t.

$$
f(t)=h_{1}(t), \ldots, h_{r}(t) \bmod P
$$


Proof of the solution's correctness: Suppose in $K_{P}[t]$ there is the factorization of $f(t)$ :

$$
f(t)=f_{1}(t) \ldots f_{s}(t)
$$

where $f_{1}(t), \ldots, f_{s}(t) \in K_{P}[t]$ are distinct monic irreducible polynomials. By the famous Hensel's lemma [13] (and $O_{K} / P$ is the residue class field of $K_{P}$ ) it follows that $r=s$ and $h_{i}(t)=f_{i}(t) \bmod P$ for $i=1, \ldots, r$ and since $h_{i}(t)$ 's and $f_{i}(t)$ 's are all monic, we have $\operatorname{deg} f_{i}(t)=\operatorname{deg} h_{i}(t)$.

In addition, under the condition $P \nmid \sharp\left(O_{L} / O_{K}[\alpha]\right)$ we have $[23,24]$ :

$$
P O_{L}=M_{1} \ldots M_{r}
$$

where each prime ideal factor $M_{i}=\left(P, h_{i}(\alpha)\right)$ in $O_{L}$. In particular, each $M_{i}$-adic local field $L_{M_{i}}=K_{P}[t] /\left(f_{i}(t)\right)$ and $\left[L_{M_{i}}: K_{P}\right]=\operatorname{deg} f_{i}(t)=\operatorname{deg} h_{i}(t)$.

Remark on Complexity of the Polynomial Factorization Algorithm for (5.1):

(5.1) can be solved via lots of algorithms, for example, the algorithm in [24] is a good solver which time-complexity is polynomial in the degree $n$ and the number of basic arithmetic operations in the (finite) field $O_{K} / P$. It's also an elegant random algorithm which successful probability is at least $4 / 9$.

Solution to the 2nd sub-problem:

$$
\begin{gathered}
\text { For any } y(t) \in K_{P}[t] /(f(t))=K_{P} \otimes_{K} L, \text { set } \psi(y)= \\
\left(\psi_{1}(y), \ldots, \psi_{r}(y)\right) \text { where } \\
\psi_{i}(y(t)) \equiv y(t) \bmod f_{i}(t)
\end{gathered}
$$

Proof of the solution's correctness: By $K_{P} \otimes_{K} L=K_{P} \otimes_{K} K[t] /(f(t))=K_{P}[t] /(f(t))$ and (5.2), it follows from the Chinese Remainder Theorem that there is isomorphism

$$
K_{P} \otimes_{K} L \cong K_{P}[t] /\left(f_{1}(t)\right) \oplus \ldots \oplus K_{P}[t] /\left(f_{r}(t)\right)=L_{M_{1}} \oplus \ldots \oplus L_{M_{r}}
$$

where the $K_{P}$-linear isomorphism $\psi$ 's components $\psi_{i}(y(t)) \equiv y(t) \bmod f_{i}(t)$, $i=1, \ldots, r$ and obviously $\psi_{i}(y \pm z) \equiv \psi_{i}(y) \pm \psi_{i}(z), \psi_{i}(y z) \equiv \psi_{i}(y) \psi_{i}(z)$ in $L_{M_{i}}$ for any $y=y(t), z=z(t)$ in $L_{M_{i}}$ and $i$. Furthermore, $T(y) z \equiv y z=$ $y_{1} z_{1} \oplus \ldots \oplus y_{r} z_{r}=T\left(y_{1}\right) z_{1} \oplus \ldots \oplus T\left(y_{r}\right) z_{r}$, i. e., there is always the diagonalization $T(y)=T\left(y_{1}\right) \oplus \ldots \oplus T\left(y_{r}\right)$ so (3.20) holds (but (3.20b) is not needed). In particular, each $\psi_{i}$ 's restriction on $L$ can be computed by:

$$
\psi_{i}(y(t)) \equiv y(t) \quad \bmod h_{i}(t), \text { for any } y(t) \in K[t] /(f(t))=L
$$

and $\psi_{i}(L)=L_{i}$.

\subsection{Solving Subproblems in Step $\sharp 3$}

Given $L$, unramified prime ideal $P$ in $O_{K}$ and local fields associated with $P O_{L}$ 's all prime factors $M_{1}, \ldots, M_{r}$ in $O_{L}$, 1.e., $L_{M_{1}}, \ldots, L_{M_{r}}$ each with an irreducible polynomial $h_{i}(t)$ in $K[t]$ s.t. $h_{i}(t)=f_{i}(t) \bmod P$ and $L_{M_{i}}=K_{P}[t] /\left(f_{i}(t)\right)$, integers $f_{1}, \ldots, f_{r} \geq 1$, we need to find $K$ 's extended fields $L_{1}, \ldots, L_{r}$ and field embeddings $\varphi_{1}, \ldots, \varphi_{r}$ satisfying (4.2). We solve this for each $i=1, \ldots, r(r \leq n)$ so the sub-problem is re-specified as: 
Given $L$, unramified prime ideal $P$ in $O_{K}$ and a local field associated with one of $P O_{L}$ 's prime factor $M$ in $O_{L}$, i. e., $L_{M}$ with an irreducible polynomial $h_{M}(t)$ in $K[t]$ s.t. $h_{M}(t)=f_{M}(t) \bmod P$ and $L_{M}=K_{P}[t] /\left(f_{M}(t)\right)$ of extension degree $f \geq 1$, find $K$ 's extended (global) field $L^{*}$ of degree $\left[L^{*}: K\right]=\left[L_{M}: K_{P}\right]$ and a field embedding $\varphi_{M}: \psi_{M}(L) \rightarrow L_{M}$ where $\psi_{M}$ denotes the $\psi$ 's componentmapping on $L_{M}$ s.t. $\operatorname{Tr}_{L^{*} / K}\left(\varphi_{M} \psi_{M}(y)\right)=\operatorname{Tr}_{L_{M} / K_{P}}\left(\psi_{M}(y)\right)$ for any $y$ in $L^{*}$.

$$
\text { Set } L^{*} \equiv K[t] /\left(h_{M}(t)\right) \text { and } \varphi_{M}=i d \text {. }
$$

Proof of the solution's correctness: Obviously $L^{*}$ is global because $h_{M}(t) \in K[t]$. Now prove $L^{*}$ is dense in $L_{M}$ and $\left[L^{*}: K\right]=\left[L_{M}: K_{P}\right]$. Since $L_{M}=$ $K_{P}[t] /\left(f_{M}(t)\right)$ is a unramified (local field) extension with extension degree $f=$ $\operatorname{deg} f_{M}(t)$ and $f_{M}(t)$ is irreducible in $K_{P}[t]$ with leading coefficient $1, h_{M}(t)=$ $f_{M}(t) \bmod P$ so by Hensel lemma $h_{M}(t)$ is irreducible in $\left(O_{K} / P\right)[t]$ with the same degree $f$ and leading coefficient 1 . In consequence[13, Chapter 14; 16, Chapter2], this unramified extension $L_{M} / K_{P}$ induces a finite field extension $O_{L} / M=\left(O_{K} / P\right)[t] /\left(h_{M}(t)\right)$ of the same degree $f$ and vice versa, a one-to-one correspondence up to isomorphism.

As a result, we have $K_{P}[t] /\left(f_{M}(t)\right)=K_{P}[t] /\left(h_{M}(t)\right)$ and in particular $h_{M}(t)$ is irreducible in $K[t]$ so $\left[L^{*}: K\right]=f=\left[L_{M}: K_{P}\right]$. By definition $L^{*}=$ $K[t] /\left(h_{M}(t)\right)$ we have that $L_{M}$ is densely contained in the field $K_{P}[t] /\left(h_{M}(t)\right)=$ $K_{P}[t] /\left(f_{M}(t)\right)=L_{M}$. Furthermore, $K_{P} \otimes_{K} L^{*}=K_{P}[t] /\left(h_{M}(t)\right)=K_{P}[t] /\left(f_{M}(t)\right)=$ $L_{M}$ so $\operatorname{Tr}_{L^{*} / K}(y)=\operatorname{Tr}_{L_{M} / K_{P}}(y)$ for any $y$ in $L^{*}$ by $(3.19)$.

Finally, $\psi_{M}(L)=L^{*}$ so $\varphi_{M}=i d$.

Remark: If $L$ is contained in $R$, so is $L^{*}$. In fact, $L$ is real so for $L$ 's any prime ideal $M, \sqrt{-1}$ is not in the $M$-adic completeness of $L$, i. e., $\sqrt{-1}$ is not in $L_{M}$. As a result, $\sqrt{-1}$ is not in $L^{*}$ which is dense in $L_{M}$, i. e., $L^{*}$ is real.

\subsection{Solving subproblems in Step $\sharp 4$}

In step $\sharp 4$ we need to solve two sub-problems. Firstly, given an ideal $A$ (with its pseudo-basis) in $L$ and a surjective homomorphism $\psi_{m}: L \rightarrow L_{m}$, compute the ideal $\psi_{m}(A)$ in $L_{m}$. Secondly the sub-problem (4.4), i. e., given $x^{*}(t)$ in $L_{m}=$ $K[t] /\left(h_{m}(t)\right)$ find $y^{*}(t)$ in $L$ s.t.

$$
y^{*}(t)=x^{*}(t) \quad \bmod h_{m}(t), y^{*}(t)=0 \quad \bmod h_{j}(t) \text { for all } j \neq m
$$




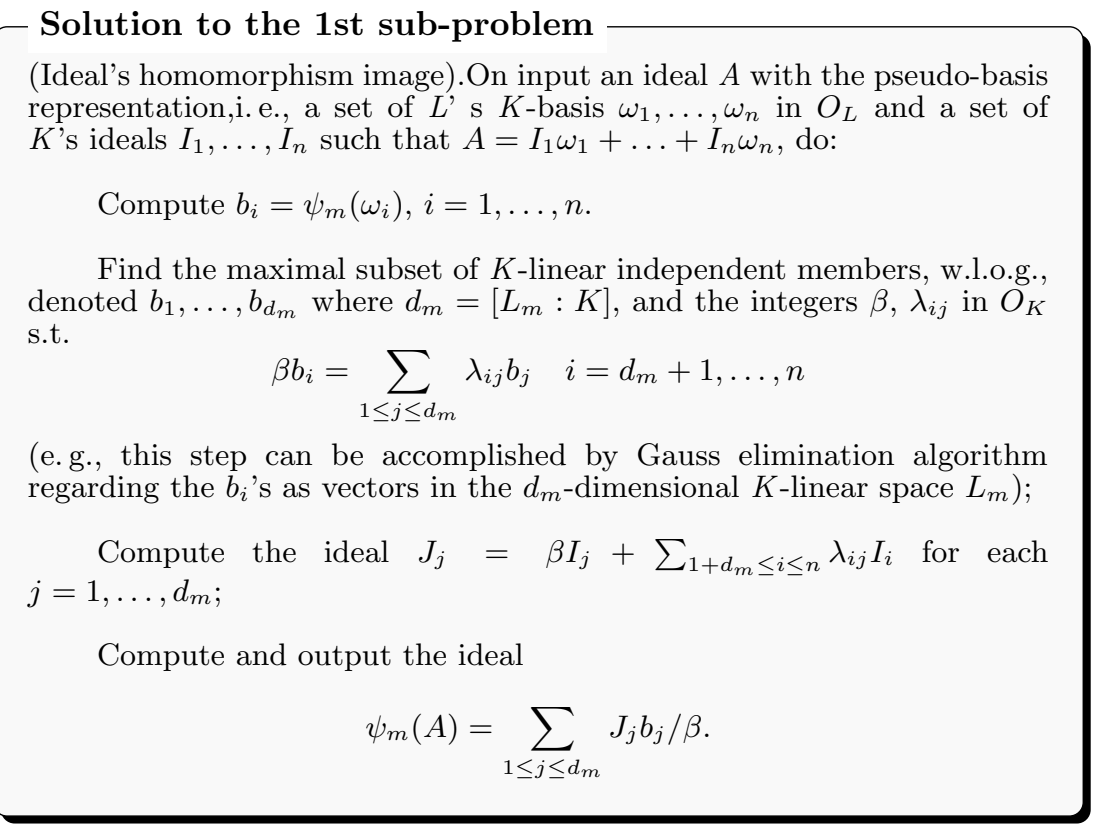

Proof of the solution's correctness: Since $A=I_{1} \omega_{1}+\ldots+I_{n} \omega_{n}$ and $\psi_{m}$ is a $K$-homomorphism, we have $\psi_{m}(A)=I_{1} b_{1}+\ldots+I_{n} b_{n}$ so

$$
\begin{aligned}
\beta \psi_{m}(A) & =\beta I_{1} b_{1}+\ldots+\beta I_{d_{m}} b_{d_{m}}+I_{d_{m}+1} \beta b_{d_{m}+1}+\ldots+I_{n} \beta b_{n} \\
& =\beta I_{1} b_{1}+\ldots+\beta I_{d_{m}} b_{d_{m}}+I_{d_{m}+1} \sum_{1 \leq j \leq d_{m}} \lambda_{d_{m}+1, j} b_{j}+\ldots+I_{n} \sum_{1 \leq j \leq d_{m}} \lambda_{n, j} b_{j} \\
& =\sum_{1 \leq j \leq d_{m}}\left(\beta I_{j}+\sum_{1+d_{m} \leq i \leq n} \lambda_{i j} I_{i}\right)=\sum_{1 \leq j \leq d_{m}} J_{j} b_{j} .
\end{aligned}
$$

and note that $b_{i}$ 's are all in $O_{L_{m}}$ since $\omega_{i}$ 's are all in $O_{L}$.

\section{Solution to the 2nd sub-problem:}

Find $g^{*}(t)$ in $K[t]$ s.t.

$$
g^{*}(t)=x^{*}(t) \quad \bmod h_{m}(t), g^{*}(t)=0 \quad \bmod h_{j}(t) \text { for all } j \neq m
$$

by the standard algorithm derived from Chinese Remainder Theorem. Then set $y^{*}(t) \equiv g^{*}(t) \bmod f(t)$

The solution's correctness can be verified by direct calculations.

\subsection{Solving Subproblems in Step $\sharp 1$}

Now we turn to this problem: given $L$ and $A$ on input, find a prime ideal $P$ in $O_{K}$ such that:

$$
P O_{L} \text { is not prime in } O_{L} \text {; }
$$




$$
\begin{gathered}
P \text { is unramified in } O_{L}, \text { i.e., all its } \\
\text { ramification indices } e_{1}=\ldots=e_{r}=1
\end{gathered}
$$

$$
P \nmid \sharp\left(O_{L} / O_{K}[\alpha]\right) .
$$

Before constructing the solver, we specify the following oracles at first.

Oracle- $P G_{K}$ where $K$ is a number field: Generates a prime ideal at random in $O_{K}$. The input is void and each output is probabilistically independent of any others.

Orcale- $P T_{L}(M)$ where $L$ is a number field: on input any ideal $M$ in $O_{L}$, tests whether $M$ is prime or not.

Oracle- $d_{K}(L)$ : On input any $L$ where $L / K$ is a number field extension of finite degree, outputs the relative discriminant $d_{L / K}$, an integral ideal in $O_{K}$ which is the greatest common divisor of $\operatorname{det}\left(\operatorname{Tr}_{L / K}\left(a_{i} a_{j}\right)\right)$ of all $K$-linear independent integers $a_{1}, \ldots, a_{n}$ in $O_{L}$.

Oracle- $l_{K}(L, \alpha)$ : On input any $L=K(\alpha)$ where $L / K$ is a number field extension with finite degree $n$, outputs $\sharp\left(O_{L} / O_{K}[\alpha]\right)$, the cardinality of the finite quotient set $O_{L} / O_{K}[\alpha]$.

\section{- Solution}

(I) Compute the relative discriminant $d_{L / K}=$ Oracle- $d_{K}(L)$; Compute $l=$ Oracle- $l_{K}(L, \alpha)$ :

(II) $\operatorname{Do}\{$ $P=$ Oracle $-P G_{K} ; /{ }^{*}$ generate prime ideal $P$ in $O_{K} * /$ \}while $\left(P \mid d_{L / K}\right.$ or $\left.P \mid l\right)$; /*equivalently, $d_{L / K}$ is a subset of $P$ or $l \in P . * /$

(III) If Orcale- $P T_{L}\left(P O_{L}\right)$ is true /*i.e., $P$ is prime in $O_{L} * /$ Then goto II; output $(P)$;

Proof of the solution's correctness By general algebraic number theory, a prime ideal $P$ in $O_{K}$ is ramified in the integral closure $O_{L}$ of the field extension $L / K$ iff it divides the relative discriminant $d_{L / K}[13,14,16]$. As a result, the output prime ideal $P$ is unramified in $O_{L}$ and obviously satisfies all other requirements in (5.7).

Remarks on implementation of the oracles: In general, how to implement all the above oracles is not completely clear with our best knowledge. However, in the important case that $L=Q(\alpha) \cong Q[t] /(f(t))$ where the polynomial $f(t)$ is monic and irreducible in $Z[t], K=Q$ (hence $O_{K}=Z$ ), we can have further arguments about their implementations.

(1) Oracle- $l_{Q}(L, \alpha)$ can be completely implemented by Oracle- $d_{Q}(L)$. If fact, in this case there is the formula

$$
\sharp\left(O_{L} / Z[\alpha]\right)=\left|N_{L / Q}\left(f^{\prime}(\alpha)\right) / d_{L / Q}\right|^{1 / 2}
$$

where $|$.$| is the ordinary absolute value.$

Proof. When $L=Q(\alpha) \cong Q[t] /(f(t))$, (due to the fact that $O_{K}=Z$ is a principal ideal domain) there exist a set of integral basis $\xi_{1}, \ldots, \xi_{n}$ s.t. $O_{L}=$ $Z \xi_{1}+\ldots+Z \xi_{n}$ and the determinant $d_{L / Q}=\operatorname{det}\left(\operatorname{Tr}_{L / Q}\left(\xi_{i} \xi_{j}\right)\right)$, i. e., $\left|d_{L / Q}\right|$ is the squared volume of the lattice $O_{L}$ 's fundamental domain. Note that $Z[\alpha]=$ 
$Z+Z \alpha+Z \alpha^{2}+\ldots+Z \alpha^{n-1}\left(\alpha \in O_{L}\right)$ is a sub-lattice in $O_{L}$ so its squared fundamental domain's volume's squared

$$
\left|\operatorname{det}\left(\operatorname{Tr}_{L / Q}\left(\alpha^{i-1} \alpha^{j-1}\right)\right)\right|=\sharp\left(O_{L} / Z[\alpha]\right)^{2}\left|d_{L / Q}\right|
$$

On the other hand, $\left|\operatorname{det}\left(\operatorname{Tr}_{L / Q}\left(\alpha^{i-1} \alpha^{j-1}\right)\right)\right|=\mid \operatorname{det}\left(\left.\alpha^{(i) j-1)}\right|^{2}=\right.$ the square of the Vandermond determinant of $\alpha$ 's conjugates $\alpha^{(1)}, \alpha^{(2)}, \ldots, \alpha^{(n-1)}=\mid \prod_{1 \leq i<j \leq n}\left(\alpha^{(i)}-\right.$ $\left.\alpha^{(j)}\right)\left.\right|^{2}=\left|f^{\prime}\left(\alpha^{(1)}\right) \ldots f^{\prime}\left(\alpha^{(n)}\right)\right|=\left|N_{L / Q}\left(f^{\prime}(\alpha)\right)\right|$, which proves (5.8).

(2) Oracle- $d_{Q}(L)$ : In this case there exist the algorithms to compute $O_{L}$ 's integral basis $\xi_{1},, \xi_{n}$ and the determinant $d_{L / Q}$, e.g., the algorithm 6.1 .8 in [25]. It's worthwhile to note that the performance-dominating step in this algorithm is to factorize the rational integer[25] which bit-size in our algorithm's context is $\operatorname{poly}(n)$, as a result, this oracle's intrinsic complexity maybe only as hard as integer factorization.

For elative extension $L / K$ where $K \neq Q$, it's worthwhile to mention the special case $d_{L / K}=O_{K}$ (which can never happen if $K$ is $Q$ ) and hence $d_{L_{j} / K}=$ $O_{K}$ for all the intermediate fields $L_{j}$ during the algorithm's recursion, e.g., $K$ 's Hilbert class field $L=K\left(\mu^{1 / q}\right)$ where $q$ divides $K$ 's class number $h(K)$. In this situation the oracle- $d_{K}($.$) is trivial and the decision P \mid d_{L / K}$ (always false) can be simply omitted from the algorithm. As a result, the algorithm's complexity can be significantly reduced (r.f., Sect.5.5).

(3) Orcale- $P T_{L}(M)$ : On input any ideal $M$ in $O_{L}$, decide whether $M$ is prime or not. For this oracle's counterpart in rational number field $Q$, i. e., rational integer's primality testing, there are not only practically efficient but also deterministic polynomial time-complexity algorithms [26, 27]. Although so far it's unknown how to efficiently implement Orcale- $P T_{L}($.$) in arbitrary number field$ $L$, it's reasonable to expect that it's complexity would be lower than SVP/CVP.

(4) Oracle- $P G_{Q}$ : Generates a prime number at random in $Z$, a problem with known efficient solvers.

\subsection{Computational Complexity}

Let $|S|$ denote the input size of the ideal lattice Problem $S V P(A, L / K), N_{P G}$, $N_{P T}, N_{d}$ and $N_{l}$ denote the number of callings to Oracle- $P G_{K}$, Orcale- $P T_{L}$, Oracle- $d_{K}$ and Oracle- $l_{K}$ in the algorithm. From the constructions in Sect.4 and Sect.5, it's easy to see that all the subroutines and operations in the algorithm are only those with time and space complexity polynomial in the input size, except the above four oracles which intrinsic computational complexity may be non-polynomial. Furthermore, the recursion depth of the (high level) algorithm is only $O(n)$ where $n=[L: K]=$ the dimension of the input ideal lattice $A$. In summary, we can have the following conclusions.

Theorem 5.1. (1) Given any number field $K$, there exists the algorithm to solve (exactly) $S V P$ on input any extended field $L$ and ideal $A$ in $O_{L}$ with time complexity

$$
\operatorname{poly}\left(n,|S|, N_{P G}, N_{P T}, N_{d}, N_{l}\right)
$$

and space complexity poly $(n,|S|)$ where $n=[L: K]$. (2)For CVP of ideal lattices, we have exactly the same conclusion. 
Corollary 5.2 (1) Given any number field $\mathrm{K}$, there exists the algorithm to solve (exactly) $S V P$ on input any extended field $L_{\mu}$ and ideal $A_{\mu}$ in $O_{L_{\mu}}$ from the family $\left\{\left(L_{\mu}, A_{\mu}\right): d_{L_{\mu} / K}=O_{K}\right\}$ with time complexity poly $\left(n_{\mu},|S|, N_{P G}, N_{P T}, N_{l}\right)$ and space complexity poly $\left(n_{\mu},|S|\right)$ where $n_{\mu}=\left[L_{\mu}: K\right]$. (2)For CVP of ideal lattices, we have exactly the same conclusion.

Remark: It is known that there exists the infinite family (e. g.the Hilbert class field extension tower) $\left\{\left(L_{\mu}, A_{\mu}\right): d_{L \mu / K}=O_{K}\right\}$ which extension degree $n_{\mu}$ is upper-boundless. For such input family, the algorithm constructed in this paper would be efficient (polynomial in time) as long as the Oracle- $P G_{K}$, Orcale- $P T_{L}$ and Oracle- $l_{K}$ can be implemented efficiently, which possiblity seems positive.

Now back to the case of $L / Q$, because there exist efficient algorithms to implement Oracle- $P G_{Q}$, i. e., to efficiently generate prime integers, we have:

Corollary 5.3 (1)There exists the algorithm to solve (exactly) SVP on input any number field $\mathrm{L}$ and ideal $\mathrm{A}$ in $O_{L}$ with time complexity

$$
\operatorname{poly}\left(n,|S|, N_{P T}, N_{d}, N_{l}\right)
$$

and space complexity poly $(n,|S|)$ where $n=[L: K]$. (2)For CVP of ideal lattices, we have exactly the same conclusion.

\section{Conclusions and Future Works}

We construct an innovative SVP(CVP) solver for ideal lattices in case of any relative extension of number fields $L / K$ of degree $n$ where $L=K(\alpha)$ is real. By this construction, solving SVP/CVP of ideal lattices is efficiently reduced to solving SVP/CVP of strictly lower dimensional ideal lattices and the problems of generating prime ideals in the ground field $K$, testing the ideal's primality in the extended field $L$, calculating the relative discriminant $d_{L / K}$ and the cardinality of $O_{L} / O_{K}[\alpha]$. The solver's space-complexity is polynomial and its time-complexity's explicit dependence on the dimension $n$ is also polynomial.

As a result, the first open problems are to construct the algorithms to implement the above oracles, which also have independent values in theory and applications. The second and more interesting open problem is that, for some of the oracles computationally hard to implement, whether its hardness can be stil1 preserved against the quantum computing model. An answer to this problem will imply whether the ideal lattice problems' hardness is solid for post-quantum cryptography. 


\section{Bibliography}

[1] Sloane, N.J., Conway, J., et al.: Sphere packings, lattices and groups. 3rd Ed. Springer-Verlag (1998)

[2] Gentry, C.: Fully homomorphic encryption using ideal lattices. Proc. 41st ACM STOC (2009) 169-178

[3] Hanrot, G., Pujol, X., Stehlé, D.: Algorithms for the shortest and closest lattice vector problems. Proc. IWCC (2011)

[4] Micciancio, D., Goldwasser, S.: Complexity of Lattice Problems: a cryptographic perspective. Kluwer Academic Publishers, Boston, Massachusetts (2002)

[5] Nguyen, P.Q., Valle, B.: The LLL algorithm: survey and applications. Springer-Verlag (2009)

[6] Micciancio, D., Voulgaris, P.: A deterministic single exponential time algorithm for most lattice problems based on voronoii cell computations. SIAM J. Comput (2012) Special Issue on STOC'2010.

[7] Kannan, R.: Minkowski's convex body theorem and integer programming. Mathematics of operations research 12(3) (1987) 415-440

[8] Ajtai, M., Kumar, R., Sivakumar, D.: A sieve algorithm for the shortest lattice vector problem. In: Proceedings of the thirty-third annual ACM symposium on Theory of computing, ACM (2001) 601-610

[9] Ajtai, M., Kumar, R., Sivakumar, D.: Sampling short lattice vectors and the closest lattice vector problem. In: IEEE Conference on Computational Complexity. (2002) 53-57

[10] Nguyen, P.Q., Vidick, T.: Sieve algorithms for the shortest vector problem are practical. Journal of Mathematical Cryptology 2(2) (2008) 181-207

[11] Peikert, C., Rosen, A.: Lattices that admit logarithmic worst-case to average-case connection factors. In: Proc. STOC. (2007) 478-487

[12] Kannan, R.: Lattice translates of a polytope and the frobenius problem. Combinatorica 12(2) (1992) 161-177

[13] Hasse, H.: Number theory. 3rd Ed. Springer-Verlag (1969)

[14] Hecke, E.: Lectures on the theory of algebraic numbers. Springer-Verlag (1981)

[15] Ireland, K., Rosen, M.I.: A classical introduction to modern number theory. Springer-Verlag (1990)

16] Lang, S.: Algebraic number theory, 2nd ed. Springer-Verlag (1994)

17. Rotman, J.J.: Advanced modern algebra. Prentice-Hall Inc (2002)

[18] Lyubashevsky, V., Peikert, C., Regev, O.: On ideal lattices and learning with errors over rings. Springer-Verlag (2010)

[19] Micciancio, D.: Efficient reductions among lattice problems. Proc. SODA'08 (2008) 84-93

[20] Cohen, H.: Advanced topics in computational number theory. SpringerVerlag (2000)

[21] Li, W.C.W.: Number theory with applications. World Scientific Singapore (1996)

[22] Haviv, I., Regev, O.: Hardness of the covering radius problem on lattices. In: IEEE CCC'06. 145-158

[23] Pohst, M., Zassenhaus, H.: Algorithmic algebraic number theory. Cambridge University Press (1989)

[24] Roblot, X.F.: Polynomial factorization algorithms over number fields. Journal of Symbolic Computation 2002(11) 1-14 
[25] Cohen, H.: A course in computational algebraic number theory. SpringerVerlag (1993)

[26] Schoof, R.: Four primality testing algorithms. In: Algorithmic Number Theory: Lattices, Number Fields, Curves and Cryptography, Cambridge University Press (2008) 101-126

[27] Agrawal, M., Kayal, N., Saxena, N.: Primes is in P. Annals of mathematics (2004) 781-793 